\title{
COVID-19 pandemic in India: through psycho-social lens
}

\author{
Aparna Joshi ${ }^{1}$
}

Accepted: 26 November 2020 / Published online: 6 January 2021

(c) Institute for Social and Economic Change 2021

\begin{abstract}
COVID-19 pandemic is an unprecedented crisis that simultaneously affected different countries and communities across the globe. The large-scale psychosocial impact of the pandemic and the subsequent lockdown, is an experiential reality for many, in India. Despite this impact, a closer look at India's response to the pandemic shows that the two strands of health and relief measures, continue to occupy centre-stage. The psycho-social aspects of the pandemic unfortunately largely remain undocumented and unaddressed. Using experiences of iCALL, National national level psycho-social counseling counselling service and a field action project of the Tata Institute of Social Sciences, the paper throws light on the much-neglected experiential psycho-social dimensions of COVID-19 pandemic and the subsequent lockdown in India. It describes the stressors experienced by users who approached iCALL and the consequent impact; especially among those who belonged to the vulnerable sections of the society. The nature of stressors as well as the impact reported by iCALL users, clearly points out to a complex interplay among several factors at individual, interpersonal, community and structural levels. The paper then, critically looks at the merits and limitations of the mental health framework that predominated the mental health response during the pandemic with its bio-medical and individualistic undertones; and suggests an alternative framing using a 'psycho-social' paradigm that views distress as an interaction between the psychological and social worlds. The paper asserts reciprocal linkages between development and psycho-social distress; highlighting the need to consciously integrate mental health issues into the development response. In the end, it makes an appeal for an inter sectoral dialogue, integrated response and advocacy for investing in mental health and psycho-social infrastructure to effectively respond to the pandemic.
\end{abstract}

Keywords COVID-19 $\cdot$ Pandemic $\cdot$ Psychosocial $\cdot$ India

Aparna Joshi

aparna.joshi@tiss.edu

1 School of Human Ecology, Tata Institute of Social Sciences, 506, Academic Building 1, Naoroji Campus, Deonar Farm Road, Deona, Mumbai 400088, India 


\section{Introduction}

COVID-19 pandemic is an unprecedented crisis that has simultaneously affected different countries and communities across the globe. Although, first reported in China in December 2019, it has been declared as an International public Health emergency by the World Health Organisation (WHO) on 30th, 2020. The pandemic has not only created a threat to physical health, but also to psychological, emotional, relational, social and economic lives of individuals, families and communities. The present paper therefore aims to highlight the psychosocial impact of COVID-19 pandemic in India. The paper begins with citing literature on the psychosocial impact of epidemics in the past, and frameworks for conceptualising this impact. It, then views this impact specifically in the light of the current pandemic. Using experiences of iCALL, a national level psychosocial helpline initiated by the Tata Institute of Social Sciences, the paper elucidates this impact and urges for its reframing through a psychosocial lens. In the end, it suggests possible steps for initiating an effective and integrated response for addressing this psychosocial impact.

\section{Epidemics and psychosocial impact}

Emergencies and pandemics are known to lead to large scale psychosocial impact. The psychological impact may include emergency or pandemic-induced distress (e.g. fear of the virus, death anxiety, diffused anxiety which is future oriented, grief, physical isolation of individuals, families or communities leading to non-pathological distress and mental health problems in a small minority), worsening of pre-existing problems (e.g. severe mental disorder; alcohol abuse) and humanitarian aid-related problems (e.g. anxiety due to a lack of information about food distribution). The social problems on the other hand include pre-emergency social problems (e.g. poverty; economic disparities, being a member of a group that is discriminated against or marginalised; political subjugation); emergency-induced social and economic problems (e.g. drastic decline of income generation, economic crisis, family separation; disruption of social networks; destruction of community fabric, resources and trust; increased violence against women and girls); and humanitarian aid-induced social problems (e.g. undermining of community structures or community's existing support mechanisms). While emergencies are likely to affect masses, certain groups of individuals such as women, children, elderly, poor, migrants, frontline workers, marginalised and those with pre-existing vulnerabilities etc., are likely to be disproportionately affected by the emergencies (IASC 2007, 2015).

Epidemics are highly impactful health emergencies, which threaten people's lives, cause significant numbers of casualties and deaths, and lead to the fear of contagion, thus endangering the safety and normal functioning of the community. Psychosocial impact created by the epidemics can exceed the individuals' and community's management capacity, often leading to high levels of distress; the effects of which can last longer even after the outbreak has ended (Johal et al. 2016). For example, studies on psychosocial effects of outbreak of severe acute respiratory syndrome (SARS) have shown negative psychological outcomes such as higher depressive levels among those who were impacted by the pandemic (Ko et al. 2006). Literature on psycho-social impact of Ebola outbreak and Nipah virus too shows comparable trends. Survivors of these outbreaks report experiencing fear of death, stigma from community, and discrimination and violence in some cases. Contacts and the 
carers on the other hand, experience anxiety, grief and stigmatization (Van Bortel et al. 2016; Zacharias et al. 2019). Physical isolation of individuals, families or communities exposed to virus can create a further risk for psychosocial problems (IASC 2015). While majority of survivors and carers show variety of distress reactions; a small minority experiences diagnosable mental health problems. Impact on the community additionally involves effects such as stigma and/or isolation, disruption in community and cultural life, loss of trust (e.g. in health services), breaking of community fabric and loss of support or coping resources (Van Bortel et al. 2016). There is also decline of income generation within communities due to travel and work restrictions, loss of family and community members, and the collapse of businesses (IASC 2015). Internationally, it can lead to stigma, discrimination and blame towards specific communities due to fear of infection; and a decreased interaction with affected areas (Van Bortel et al. 2016).

While there is a large-scale experience of distress and universal need for psychosocial support during the epidemics, there is a varying degree of vulnerability experienced by different groups based on their age, gender, social locations; especially by those who have precarious living conditions, few resources, and limited access to social and health services. The healthcare providers and frontline workers too are at risk of experiencing high amounts of distress (Grace et al. 2005; PAHO 2016). Having looked at the psycho-social impact of epidemics, it is also important to understand frameworks for conceptualising the same.

\section{Conceptualising distress in disaster, emergency and epidemic context: conflicting frameworks and competing agendas}

There are several competing frameworks for conceptualising and responding to the mental health and psychosocial impacts of emergencies and disasters (Ventevogel 2018; Weiss et al. 2003). This section elaborates upon some of these frameworks, and their merits and demerits.

The first framework - 'trauma approach' - conceives psychological distress as normal responses to abnormal external situations (e.g. emergency, disaster, pandemic), and highlights 'psychological trauma' as the most important issue. The key response in this framework includes the fixing of 'emotional scars' and the resolution of traumatic memories using trauma specific treatment formats (Ventevogel 2018). The approach is credited for acknowledging and normalising distress, and attributing responsibility of the same to the powerful external source of trauma (emergency, crisis, pandemic etc.). And yet, it is riddled with several problems. Despite its utility in creating a shared language for distress, it has been criticised for creating an unnecessary psychiatric label for what should be considered a normal response when coping with extreme stressors; and for even stigmatising it (Weiss et al. 2003). The trauma approach by clubbing all distress experiences under one label, may flatten the description and meaning of those experiences. The categories of psychiatric disorders imported from Western psychiatry world, such as post-traumatic stress disorder (PTSD), may not appropriately capture the cultural variations of trauma experiences and meanings (Priya 2018; Weiss et al. 2003). And lastly, one of the biggest criticisms of the trauma approach to emergencies and pandemics pertains to its interference with the agenda of community-based mental health programs (Weiss et al. 2003) and instead individualising it. Psychologising or psychiatrising emotional distress into mental disorders that need 
treatment, may often lead to obscuring the driving socio-political causes of emotional distress which need interventions along with the resulting distress (Pandey et al. 2020).

The second framework-diagnostic approach or psychiatric approach, captured in international classificatory systems of psychiatric or mental disorders, involves labelling distress and categorizing people on the basis of sets of symptoms they show (Kinderman 2014). This categorisation, in turn is expected to offer a standardised language in mental health field; facilitate discussion about aetiology; and provide directions for interventions. While the diagnostic approach, informed by its underlying disease model, continues to be a predominant discourse in the mental health field, there have been several criticisms levied against it. A few of these include its failure to capture a holistic picture about causation of a psychiatric disorder, or of the person's experience of their distress; its reductionist conceptualisations of mental health, despite the tremendous heterogeneity of mental health concerns and its insufficiency in providing directions for guiding interventions (Macneil et al. 2012). The approach also ignores the wider context of a person's experience (Kinderman 2014) as well as the mounting evidence on contribution of psychological, social and environmental factors ranging from childhood adversities, family environments, social support, violence, poverty, unemployment, migration, humanitarian crisis, socio-economic disparities (Alegría et al. 2018; Burns 2016; WHO 2014) in production of mental distress. Diagnostic approach is also criticised for its treatment/intervention implications. Often standing on the shoulders of bio-medical models, it is known to strengthen pharmacological and hospital-based care, while disempowering non-medical treatments and support options (Jacob 2006); and silencing community voices and participation (Jain and Jadhav 2009). Applied specifically to postcrisis and postdisaster situations, the model is criticised for pathologizing naturally occurring distress responses (Weiss et al. 2003) and ignoring sociopolitical or cultural contexts of distress as well as the resultant fragmenting of relationships and communities (Priya 2018). One of the biggest critiques of the diagnostic approach in pandemics such as COVID-19 pertains to its disappointing omission of references to systemic vulnerabilities and social injustices that often existed in the pre-COVID contexts and were further intensified during the pandemic (Burgess 2020; Pandey et al. 2020).

The third framework-'psychosocial approach-draws from the revolutionary biopsycho-social model proposed by Engel (1977) involving the complex interplay of biological, psychological and social dimensions in the development of psychiatric disorders, and from the theoretical work around social ecologies (Bronfenbrenner 1986) that locates distressed individuals within the social context of a family or household further nested in communities and societies. The psychosocial approach, with its linage in community and peace psychology as well as human rights discourse, is widely acknowledged in the context of Humanitarian emergencies and responses (Ventevogel 2018). It blends the psychological (subjective and inter-subjective experiences) with the social (social structures of class, ethnicity, gender etc.), and employs the psychosocial context in interpreting and understanding mental health concerns (Johal et al. 2016). The psycho-social approach goes beyond conceptualising 'health' as the absence of disease or illness or symptoms; and views it as an ability (Sytema 2006). It draws from evidence that shows a link between mental health and social, economic, and physical environments in which people live; and between Social inequalities and increased risk of many common mental disorders (WHO 2014). The term psychosocial support represents 'any type of local or outside support that aims to protect or promote psychosocial well-being and/or prevent or treat mental disorder' (Inter-Agency Standing Committee). This approach includes support interventions in several sectors such as health, education, community- based interventions etc. to address a range of problems including emotional distress, socio-economic-political problems and 
mental health concerns. In contrast to the earlier cited diagnostic approach which focuses on mental health disorders, and offers individually tailored treatment packages; psychosocial interventions tend to focus more on influencing the psychosocial context and on promoting health and wellbeing (Johal et al. 2016).

Despite the criticisms of lack of vigorous documentation of outcomes and impact, and of the weak definitions of hypothesized causal pathways to wellbeing (Ventevogel 2018), psycho-social approach presents a compelling ideological and strategic alternative to the mainstream diagnostic model of understanding distress experienced during the pandemics. It acknowledges that distress is considerably more prevalent during disasters and emergencies, than mental health disorders; and, that for most people, the distress is tolerable, shortlived and dependent upon the duration of the stressors. The approach, thus, legitimises this distress as negative experiences and emotions, without pathologizing it. Psychosocial approach believes that all those involved in an emergency are likely to benefit from some form of psychosocial support; and hence, calls for holistic and integrated interventions for influencing the psychosocial contexts, and promoting health and wellbeing. At the same time, it also acknowledges that for a small minority of population, a formal or professional intervention as well as specialised mental health services may be needed as this population is seriously affected by the emergencies, or has experienced an exacerbation of the preexisting mental health problems (Weiss et al. 2003).

Mental health and psychosocial support (MHPSS), is a composite term used to describe any type of local or outside support that aims to protect or promote psychosocial wellbeing and/or prevent or treat mental disorder. Yet, it is important to remember that both these terms have different lineages. As mentioned earlier, the term mental health is largely embedded in a diagnostic framework, while the term psychosocial support refers to a range of non-biological interventions that target psychological and social factors affecting those in distress (IASC 2007). Hereafter, the paper continues to use these terms in the way described above.

After understanding the frameworks for conceptualising and responding to the distress experienced during emergencies and the pandemics, and making a case for adoption of psychosocial approach, the paper now moves on to elaborate upon the nature of psychosocial impact of COVID-19 pandemic.

\section{Mental health and psycho-social impact of COVID-19}

Being the epicentre of the disease, some of the initial evidence on mental health effects of the pandemic emerged from China. This evidence showed that COVID-19 posed an increasing risk of mental health concerns among general public, and exacerbation of symptoms among those who had a pre-existing diagnosis. Some of the commonly documented emotional impacts included anxiety, worry and depression (Zhou et al. 2020). Younger people and healthcare workers were found to be at a greater risk for developing symptoms of mental illness (Huang and Zhao 2020). Literature reviewing mental health impact from other parts of the globe, slowly showed similar trends and reported a number of individual and structural variables that moderated this risk (Rajkumar 2020).

The first case of COVID-19 in India, was reported in January 2020. The pandemic slowly created a sense of fear and panic in the Indian society. Since 24th March, the Indian Government took a series of actions to contain the spread of the virus. Unfortunately, these actions involving long and strict lockdowns of the entire population without adequate 
warning and protection, resulted in serious psycho-social distress. The large-scale psychological impact of the pandemic and the subsequent lockdown, was an experiential reality for many in India (Varshney et al. 2020), signalling onset of a psychosocial crisis.

As mentioned earlier, literature on the psychosocial impact of emergencies shows that while most people affected by an emergency experience some level of distress, specific vulnerable sections of the society (those groups who disproportionally experience adverse impact of the emergency due to their disadvantaged social locations) are likely to experience greater distress (Johal et al. 2016). The COVID-19 pandemic too posed a serious threat to the psychosocial well-being of some of the disadvantaged and vulnerable groups in India. For example, internal migrants in India reported adverse psychosocial impact of the pandemic (Choudhari 2020). Several other vulnerable groups such as individuals diagnosed with COVID-19 or those in quarantine, children, women survivors of violence, elderly, people with prior history of mental illness, LGBTQI individuals and frontline workers experienced intense distress. Health Care providers reported heightened symptoms of anxiety and stress (Chatterjee et al. 2020). Noticing these trends, mainstream news media announced arrival of an emerging mental health crisis in India (Moses 2020; Sudan and Narayan 2020). It was also acknowledged that some of these negative psychosocial effects were not just transitory, but capable of creating a long-term impact on individuals and society.

While the importance of psychosocial services is evident during such times, the traditional face to face services may not be available due to the pandemic, and the subsequent containment strategies. Remote support services, that are technologically assisted (previously considered to be a peripheral), thus become important during the pandemic (Malathesh 2020; Rosen et al. 2020). Using iCALL helpline's experiences, the paper now elucidates the nature of psychosocial concerns and impacts experienced by individuals in India during early phases of the pandemic.

\section{Methodology}

\section{The context}

iCALL is a national level technology assisted psycho-social counseling service and field action project of the Tata Institute of Social Sciences, India. iCALL was established in September, 2012 with an objective of providing accessible, affordable and quality counselling services to individuals in distress; particularly to those who belong to the disadvantaged and vulnerable communities. iCALL is a telephonic, email and chat-based facility which offers counselling services to individuals in distress across the lifespan. This multilingual service (10 different languages) is run by trained professionals, and is free, confidential and anonymous. Apart from providing psycho-social counseling services, iCALL also engages in other activities such as research, capacity enhancement of mental health and helping professionals and outreach, and awareness creation on mental health and allied issues.

During the COVID-19 pandemic, iCALL continues to offer its services through its regular telephone line as well as through a dedicated line addressing psycho-social impact of COVID-19 pandemic. iCALL's COVID line aims to assist individuals and communities by providing safe space for addressing emotional distress, reliable information and referral linkages to for health, livelihood, food, transportation and other areas of life. The service 
is conceived as an important effort to demonstrate how social, practical and psychological aspects of COVID-19 pandemic can be addressed in an integrated fashion.

iCALL adopts a non-pathological, diversity friendly, strengths based and psycho-social lens to conceptualise and respond to distress. iCALL believes that an effective and sensitive response to distress warrants not only an emotional and therapeutic support, but also access to resources and control over the determinants of mental health (livelihood, food, shelter, safety, resources, access to health facilities etc.). This involves simultaneous engagement with individuals' psychological and social worlds. The psychological interventions include provision of a safe and empathetic listening space, containing callers' emotions and building awareness about the same, exploring ways of dealing with negative emotions, behaviours and cognitions; increasing sense of safety, providing information, and connecting clients to their strengths, internal resources, past healthy ways of coping and support systems. At the same time the counselling interventions go beyond the traditional ones that often focus only on intrapsychic and individual factors. Given the fact that the distress reported by the callers largely emanates from the economic crisis, loss of livelihood and resources, loss of support, mobility restrictions, state policies, patchy relief work and violence, hostility and stigma, iCALL actively links the callers to practical support, resources and referrals at the ground level. The distress experienced by callers is not perceived as individual failure; but reframed in the light of the structural constraints that contribute to this distress. Counselling conversations then are viewed as spaces for individuals to voice their dissent and resistance against larger systemic oppression; and discuss ways of negotiating power. Each call ends with the counsellor seeking feedback from the caller to ascertain if the caller felt heard and helped. The feedback from callers received so far, has been encouraging, and assuring iCALL of the directions assumed.

\section{Procedure}

The paper is based on a preliminary analysis of calls received by iCALL dedicated COVID19 helpline during the first month of its functioning. The helpline received a total of 349 genuine calls during its first month after the launch (April 18th to May 18th). Each of these calls was documented by the counsellors in the specially designed documentations format. Given iCALL's stance on maintaining the anonymity and confidentiality of the callers, data regarding demographic details was captured only if (and as) shared by the callers. Counselors documented details of the concerns reported by the callers and the interventions provided. While callers often presented with multiple concerns, calls were categorised into a pre-defined format, based on the predominant concerns reported by the callers. The quantitative data was analysed using descriptive statistics, while the call transcripts were thematically analysed (Braun and Clarke 2006) to draw insights about predominant concerns raised by the callers and the psychosocial impact of the same.

\section{Results}

The following section first presents the profile of the callers and then moves on to elaborate upon some of the themes describing the nature of concerns reported by the callers and the resultant psycho-social impact.

As can be seen in Table 1, majority of the calls were from male callers and from young adults. This is not surprising given the issue of access to telephone and privacy in the 
Table 1 Profile of the callers

\begin{tabular}{|c|c|c|c|}
\hline S no. & Caller characteristics & Frequency & Percentage $(\%)$ \\
\hline \multirow[t]{3}{*}{1.} & $\operatorname{Sex}$ & & \\
\hline & Male & 235 & 67.5 \\
\hline & Female & 113 & 32.5 \\
\hline \multirow[t]{6}{*}{2.} & Age & & \\
\hline & $18-30$ & 138 & 39.7 \\
\hline & $31-40$ & 55 & 15.8 \\
\hline & $41-60$ & 25 & 7.2 \\
\hline & 61 and above & 2 & 0.6 \\
\hline & Age not known & 128 & 36.8 \\
\hline \multirow[t]{11}{*}{3.} & Callers' concerns & & \\
\hline & Helpline information & 49 & 14.1 \\
\hline & Academic and career related concerns & 20 & 5.7 \\
\hline & Relationship concerns & 19 & 5.5 \\
\hline & Health concerns & 22 & 6.3 \\
\hline & $\begin{array}{l}\text { Practical concerns (food, transportation, essen- } \\
\text { tial services etc.) }\end{array}$ & 107 & 30.7 \\
\hline & Livelihood and financial concerns & 31 & 8.9 \\
\hline & Violence against women & 9 & 2.6 \\
\hline & Emotional distress concerns & 46 & 13.2 \\
\hline & Suicidality & 6 & 1.7 \\
\hline & Other & 39 & 11.2 \\
\hline
\end{tabular}

$N=348$

Indian communities, favouring men and young adults. Given the early days of the pandemic, many callers $(14.1 \%)$ called the helpline to test its credibility and also to understand the nature of help provided, followed by those who needed practical support (30.7\%) and help for addressing emotional distress (13.2\%).

Table 2 Callers' concerns: themes and sub-themes

1. Psychosocial concerns among general public

1. The pandemic and the consequent Fear

2. Disappointment with the health system

3. Economic and professional worries, practical concerns, and disruption of life plans

4. Psychological and emotional distress

5. Family dynamics and increased conflicts

6. Troubled intimacies

2. From the margins: vulnerable groups and psychosocial impact:

1. Psychosocial impact: COVID patients and health workers

2. Migrant workers and psychosocial impact

3. Women survivors of violence and abuse

4. Individuals with Prior mental health vulnerabilities

5. LGBTQ individuals and psychosocial impact 
The following table presents themes and subthemes that emerged from the analysis of call transcripts (Table 2).

\section{Psychosocial concerns among general public}

\section{The pandemic and the consequent fear}

One of the initial impacts reported by iCALL callers was related to the fear of the pandemic. The disease itself created a sense of panic and anxiety (including death anxiety) in people's minds. Low levels of awareness about the disease, its changing symptomatology and its novel nature, caused further anxiety among public; especially during the initial stages. Callers reported experiencing worry towards possible signs and symptoms of COVID-19 in themselves and their loved ones. They approached iCALL with several queries related to signs and symptoms of the disease, and prevention measures to be undertaken.

The media plays a significant role during emergencies and epidemics by informing people about the situation and keeping them updated on measures taken by governments to contain the spread of the virus (IOM 2020). Research findings show that too much of media exposure can be harmful to mental health; with social media escalating anxiety more than the traditional media (Weir 2020). Strong sets of emotions stirred up by the media, may lead people to judge risk by these emotions rather than facts and data (Weir 2020). Similar trends were seen among iCALL callers. Those who had access to audio-visual, digital and social media were bombarded with information, which often relayed ill-founded and contradictory messages. Constant exposure to reports of rising number of cases and deaths, affected people's psyches, creating an atmosphere of terror and threat. Individuals were worried not only about their own safety, but also of their loved ones.

\section{Disappointment with the health system}

Adequate and credible health system can play an important role in addressing and alleviating some of these anxieties faced by the general public. These include measures such as raising awareness and providing reliable information about risk and potential health consequences of the virus as well as progression of a pandemic, and prevention and intervention measures; ensuring adequacy of testing and medical facilities; making available health personnel and protection equipment; enacting steps to reduce the spread of the virus in the community and in health-care facilities; setting up of tele outreach programmes to address queries and to provide telehealth consultation; protect and support health-care workers during a pandemic etc. (WHO 2009).

However, during the COVID-19 pandemic in India (barring a few noteworthy examples), the health system did not perform adequately to address the pandemic effectively and inspire confidence in people (Chatterjee 2020). iCALL callers shared painful, disappointing and anxiety provoking experiences of dearth of testing facilities and of credible health services, delayed reports, and negligence (and at times exploitation) at the hands of the health system. iCALL callers expressed their frustrations over lack of availability of beds and health facilities. Some also complained about being overcharged for medical facilities, tests and hospital stay. The public health system in India, is known to be riddled with several inadequacies including those related to poor financing, dearth of accessible 
and affordable health care services, inadequate human resources and poor accountability (Kasthuri 2018). The pandemic further burdened the struggling health system, resulting in diversion of its resources to the treatment and prevention of COVID-19, and in making regular health services inaccessible to common public. Changes such as mobility restrictions and lack of transportation leading to compromised access to healthcare, inadequate supplies of medicines, shift to telemedicine and online practices, further added to the plight of the individuals.

\section{Economic and professional worries, practical concerns, and disruption of life plans}

Due to the lockdown and restrictions on mobility, callers approached iCALL to seek assistance for practical concerns related to food, medicines, transportation, and health and essential services. Callers also approached iCALL with several concerns related to livelihood and employment. They reported anxiety and uncertainty about an actual or possible job loss, pay cut, pending bills, inability to buy food, inability to pay EMIs etc.

The disease and subsequent lockdown disrupted life plans that were taken for granted by many individuals; especially by students, employers, employees etc. The sudden changes in school and college schedules and particularly in exam timetables, created an extreme sense of uncertainty and panic among students (John 2020). Those who were planning for future careers, had to change or forgo their life plans. There were many others who had planned important life events such as marriage, job transfers, buying a new house, travel, surgeries etc.; all of which had to be significantly altered. This created a huge sense of disruption and a loss of control.

Employees working in organised sector reported having to bring their offices into their homes. Initially, 'work from home' appeared as a positive prospect, especially to those who lived in metro cities, as it provided the necessary respite from long commute and hectic work hours. Employees looked at this as an opportunity for increased productivity. However, as the lockdown got extended, individuals reported contrary experiences of low productivity and lack of motivation. They started complaining about fatigue caused by prolonged screen interface, lack of differentiation between personal and professional lives, dearth of human interactions with colleagues, lengthy communication loops to get simple tasks done etc. Those individuals who considered their professional lives (and performance related to the same) as central to their identities, found it most difficult to accept possible losses, uncertainties and failures. Women were doubly affected as they lost their own jobs and/or those of their partners who were the primary bread winners. Competing home and work demands exposed women to further risks of pay-cuts or lay-offs.

\section{Psychological and emotional distress}

Callers approaching iCALL reported myriad of negative emotions such as feeling anxious, sad, bored, lonely, a sense of loss and grief, anger, frustration etc. Many also reported a feeling of numbness and a disconnect from every day reality. Some complained about the fear of losing every day life skills during the lockdown. Despite the talk of the 'new normal', the reality of the normal remained dynamic and illusive, leaving many with a sense of loss of control over their own lives. Callers complained about their depleting internal and external coping resources as the lockdown got repeatedly extended. The older ways of coping with distress, which were often linked with participation in the outside world (e.g. talking to friends or neighbours, visiting religious places, going for a walk, going for a movie, 
shopping etc.) were inaccessible due the lockdown. While some quickly adapted to, and developed newer ways of coping with the changed reality, others struggled to do so. For some, this also led to existentialist questioning about the meaning of life and death.

\section{Family dynamics and increased conflicts}

One of the significant themes for which callers repeatedly sought help from iCALL, pertained to relationship conflicts and their psycho-social impact. These conflicts were reported by individuals across different age groups and in several kinds of relationships including (but not limited to) parent-child, intimate, married, long distance, peer relationships etc.

Indian families, often described and boasted for their collectivistic nature, when forced to stay together for extended periods, found it difficult to navigate through this enforced togetherness. The lockdown disrupted everyday rhythms of family functioning, and robbed people of the much-needed distraction provided by work, schools and outside life. The lockdown brought 'hierarchical and unequal nature' of the family systems in India, to the forefront. Adolescents and young people found it restricting and frustrating to be monitored by their parents and elders, and to be sermonized on issues pertaining to screen time, digital dependence and household chores. Parents on the other hand reached out to iCALL to seek tips for keeping their children engaged at home, and for managing anger outbursts and conflicts in their teenagers.

Literature shows that the multifaceted effects of the pandemic have been exacerbated for women and girls (Mathews 2020). Given the traditional gender role expectations of caregiving from women, and unavailability of house help during the lockdown, women felt completely unsupported by the family members as they conducted the household responsibilities. Women complained about the exponential increase in unpaid care work with school closures and heightened care needs of family members at home. They also expressed their frustration and anger about the unequal and unfair division of labour at homes.

Literature also shows that measures such as social distancing may have grave consequences for the mental health of the elderly, leaving many in a state of isolation and depression (2020) and contributing to a possible increase in elderly suicides during the pandemic in India (Rana 2020). iCALL too was approached by some older adults who were separated from their children and loved ones, and needed practical as well as emotional support. On the contrary, there were a few others, who were forced to live with their children during the pandemic, and found it difficult to accept their increased dependence on their younger children and the resultant loss of freedom and dignity. iCALL also received calls from adult children of the elderly who played care-giving roles for their older parents. These individuals reported worry for the health of the elderly, and burn-out and exhaustion due to the constant caregiving burden. Some also called for psychiatric referrals to address relapse of mental illnesses in their elderly parents.

\section{Troubled intimacies}

Murray Bowen's family systems theory is one of first comprehensive theories of family systems functioning which proposes that families attempt to balance two life forces-family togetherness and individual autonomy-, that one of the known sources of conflicts in intimate relationships is lack of balance between togetherness and separateness (Bowen 1978). This core conflict reflected in the calls that surrounded issues of intimate relationships, 
woven around both ends: 'frustration due to forced togetherness' and 'isolation due to separation from the loved ones'.

iCALL was approached by many who were stranded in the same household with their partners for months together without an escape; making the experience frustrating and distressing. Many reported that the old conflicts, which were supposedly buried under the carpet, re-emerged during the lockdown; a phenomenon described as the 'return of the repressed' (Veliyannoor 2020). The usual household decisions around food, routine, work, expenditure, habits etc. became grounds for contestation. Individuals also reported newer triggers for conflicts surrounding issues such as quarantine, preventive measures, testing, stepping out of the house, travelling or inviting visitors at home. Callers complained that their earlier strategies of conflict resolution in intimate relationships, failed in these circumstances, often leaving them hurt, frustrated and sad. While some reported forced sharing and intimacy as a source of conflicts, others also reported that isolation and social distancing from partners and family members, created negative consequences for their mental well-being.

\section{From the margins: vulnerable sections and psycho-social impact}

While COVID-19 pandemic is perceived and proclaimed to be an equaliser in bringing out vulnerabilities in different strata and sections of the society, it also proved to intensify the already existing structural inequalities in the Indian society. The following section closely looks at the psycho-social impact of COVID-19 pandemic on those individuals who belonged to the vulnerable sections of the society.

\section{Psychosocial impact: COVID patients and health workers}

Literature points out to special mental health vulnerabilities among those who are quarantined (Chatterjee and Chauhan 2020) and also among the healthcare and frontline workers (Spoorthy et al. 2020). Callers who were in quarantine at home and in health facilities, reported sense of dejection, disconnect, anxiety, and also experiences of stigma and trauma. Given their constant exposure to the health risk, many reported anxieties (including death anxiety) and concern about their own health and also of their loved ones.

Stigma is a major social determinant of health that drives morbidity, mortality, and health disparities (Hatzenbuehler et al. 2013) and has been described by the World Health Organization as a 'hidden' burden of disease (2001). Stigma against patients and health care providers during COVID-19 pandemic, and its mental health effects are slowly being documented (Singh and Subedi 2020). iCALL received a few calls that mentioned the stigma faced by COVID positive patients as well their family members. The infectious nature of COVID-19 disease, along with preventive measures of lockdown and containment, and stigma particularly targeted at certain communities (caste, class and religion), created an atmosphere of suspicion and doubts among Indian communities. Words like 'hotspot', 'COVID-suspects', criminalised and blamed those who were the victims of the pandemic. Strategies such as contact tracing and marking COVID-19 affected areas with identifiable symbols, further added to the differential treatment and stigma experienced by those who were infected with COVID-19. Family members of patients too were targets of hate and discrimination. Small number of calls from the healthcare providers and individuals involved in provision of essential services as well as frontline workers, too, reported 
stigma faced by them, and the threats of being evicted from their households (Yeung and Gupta 2020). Some called us to explore referral sources for procuring personal protective equipment and also for reporting the hazardous conditions in which they worked.

\section{Migrant workers and psycho-social impact}

Internal migrants are among the most susceptible communities in India, often facing several physical and mental health challenges (Raghavan and Cooper 2018). Calls received from migrant workers during the COVID-19 pandemic were one of the stark examples of how the pandemic, actually intensified the existing rifts within the social structures in India.

Being an outsider to the host city and culture, already makes migrants a vulnerable community. The pandemic and the subsequent lockdown led to further intensification of uncertainty and economic vulnerability among the migrants, as many lost their livelihoods and sources of income. The sudden lock down, coupled with loss of income made it difficult for migrants to continue to live in the cities. High prevalence of COVID-19 cases, and the fear of contracting the disease itself, heightened their anxieties. The city became more and more inhospitable, further intensifying a sense of panic and alienation among migrants; leading to desperation to return to their homelands. Lack of access to information, transportation, income, housing, food, health and essential facilities as well as support systems, worsened the plight of migrants in the metro cities. Many of them reached out to iCALL for assistance with these concerns.

Male migrants reported being locked into their tiny rented houses with their colleagues, surfing on their mobiles for the whole day, and worrying for their loved ones who were back home. Traditional Masculinity scripts of being providers and bread winners often prevented male migrants from sharing their vulnerabilities with the family members back home. iCALL also received calls from family members of the migrants. Family members back home too were worried about the safety of their migrant relatives who lived in cities which were breeding grounds of COVID-19 infection as portrayed in the media. Some of the male migrants who approached iCALL expressed a sense of helplessness and desperation during their calls, while many others expressed intense anger against the system. This also included anger towards police personnel who showed complete apathy when were approached for help. Migrant workers did not know what the future held for them as they felt unwelcomed by the city and cut off their families and communities back home.

\section{Women survivors of violence and abuse}

National Commission for women in India, during the initial phases of the lockdown itself, publicly acknowledged that the number of domestic violence cases in the country had continued to increase (Roy 2020). Some of the pathways that increase women's risk to violence during the pandemic include amplified household stress, disruption of livelihoods, reduced access to health and essential services, restricted access to money, disruption of social and protective networks and restricted connect with formal support services such as hotlines, crisis centres, shelters, legal aid, and protection and counselling services (Roesch et al. 2020).

iCALL too was approached by a few girl and women survivors of abuse and violence. They reported exacerbation of violence that existed before the pandemic. Apart from the everyday violations experienced by girls and women within the household spaces, 
violence during the pandemic assumed some newer manifestations. Women reported that men and other family members who perpetrated violence, adopted newer 'scare tactics' that involved misinforming them about the pandemic, and creating a sense of fear for their own lives or those of their loved ones. Violence also took the form of depriving women of resources such as soap, sanitisers, masks that were important in protecting them from the virus. Women were often the last ones to receive health care and relief services.

The economic crisis and loss of livelihood directly affected those women who were earning members, further increasing their economic dependence on the male members within the family. Women also experienced an indirect impact of this economic crisis by becoming displaced targets of the anger and frustration experienced by their male members who lost their livelihoods. Attempts to assert their own needs or to negotiate power and responsibilities within the household, were often met with further violence. Being stranded with perpetrators of violence who had total access to their mind, labour and bodies, increased women's vulnerability, and aggravated the general anxiety and panic created by the pandemic. Withdrawal symptoms experienced by their partners who could not access substances, became excuses for the violence hurled at them. Limited possibilities of escape due to mobility restrictions and lack of access to support structures; heightened women's sense of terror and helplessness. Given the patriarchal control over mobile and telephone devices, women had to find quiet and un-surveilled slots to call iCALL helpline and share their stories. The police and health systems, when contacted, were already flooded with pandemic related responsibilities, and hence were less responsive.

\section{Individuals with prior mental health vulnerabilities}

Individuals with prior histories of mental health concerns formed the next vulnerable group. Many reported feeling lonely and disconnected from their usual support systems. Dearth of affordable, accessible and reliable mental health services further added to the plight of those with prior mental health concerns. The mental health services that existed prior to the pandemic were face to face in nature; and hence, took time to transition into a technology assisted mode. This resulted in scarcity of treatment and support options, leaving many without renewed prescriptions and therapeutic services.

\section{LGBTQ individuals and psychosocial impact}

A statement issued by human rights experts on the International Day against Homophobia, Transphobia and Biphobia (Council of Europe 2020), specifies that LGBT children, youths and elders, during the pandemic are forced to endure prolonged exposure to unaccepting family members, which in turn is intensifying rates of domestic violence and physical and emotional abuse, as well as damage to mental health.

A few of the LGBTQI individuals, who reached out to iCALL, shared stories of being stranded with families unaccepting of their sexual and gender expressions. Some of them had moved away from their families in order to find freedom for their gender and sexual expressions. When forced to move back into the familial spaces, these individuals once again found themselves hiding their real selves. Those who had continued to live with their families without revealing their identities, found it suffocating to continue the façade. Some also reported harassment and abuse from their family members. Being cut off from their support systems, posed additional challenges for their mental health. 
Issues mentioned in the above sections, and concerns faced especially by individuals belonging to the vulnerable groups, bring to the forefront the multifaceted nature of distress, and also its embeddedness in larger structural inequalities and injustices that existed in the pre-COVID era. Having closely looked at the stressors experienced by the general public and vulnerable and marginalised groups, and their psycho-social impact; it is imperative to look at the measures taken by the Government as well as civil society organisations in India to address this impact. The next section throws light on India's mental health and psycho-social responses to the pandemic, and suggests an alternative farming through a psycho-social lens.

\section{Mental health framing and response to the pandemic in India}

With the slow, but growing acknowledgement of the mental health impact of the pandemic, arose a question of India's capacity to handle this mental health crisis (Sahoo and Biswal 2020). The following section traces India's mental health care system prior to the pandemic, and response during the pandemic.

\section{India's mental health care: prior to the pandemic, and in response to the pandemic}

Mental health has been neglected in India's health and political agenda over the years. Given the 150 million people who are in need of mental health services in India, and a treatment gap which ranges from 70 to $92 \%$ for different disorders, the Indian mental health care system was already struggling prior to COVID-19 (NMHS 2016). The National Mental health programme (NMHP), launched in 1982, aims to ensure the availability and accessibility of minimum mental healthcare for all; enhance community participation in the mental health service development; stimulate self-help efforts in the community and reduce stigma (Government of India 1982). The National mental health programme and its successor, District Mental Health Programme heavily rely upon integration of mental health services into the existing public health infrastructure, as the central strategy for making mental health services available to the masses. Despite its stated aims and commitments, NMHP has been severely criticised for several reasons ranging from uneven coverage and provision of mental health services, human and financial resource constraints, lack of comprehensive training to the care providers, poor or virtually absent community participation and its less emphasis on preventive and promotive aspects (Gupta and Sagar 2018). One of the consistent critiques against India's National mental health programme is related to its bio-medical framing of mental health issues and exclusive reliance on pharmaceutical solutions to psycho-social problems (Jain and Jadhav 2009).

The public health and economic crisis ensued by the COVID-19 pandemic, necessitated health response and relief work, which continued to occupy the centre stage of India's response. Despite the past evidence of the mental health and psycho-social impact of pandemics and emergencies, and the need for interventions to address the same (mentioned in the beginning of the paper), as well as the strong recommendation provided by United Nations policy brief (2020) to consider mental health as essential component of the National response to the pandemic, psycho-social and mental health care response remained inadequate and scattered in India (Nair 2020). Many of the development, social protection and relief measures did not consciously account for this mental health impact 
in their design; nor did they intentionally reach out to people with mental health concerns who are often known to fall through the cracks of developmental aids.

A closer look at India's response to the pandemic shows that the two strands of health and relief measures, continued to occupy centre-stage. Mental health response was a late entrant into the list and was initiated by several actors and stakeholders. Given the difficulties in provision of face to face services during the lockdown, some of the early responses from the Central and state Governments, were to initiate mental health helplines. These helplines were often started in collaboration with Academic Institutions (Ministry of Health and Family Welfare 2020), regional mental health or psychology chapters (Kumar et al. 2020), Civil society organisations or the District mental health programmes (John et al. 2020). Some of the other responses included drafting of guidelines for addressing mental health issues for at risk groups (NIMHANS and Ministry of Health and Family Welfare 2020) and offering preliminary orientation to the existing public health and mental health systems as well as to personnel involved in other programmes addressing safety and well-being of vulnerable populations such as adolescents, elderly, women etc. Needless to say, that these measures were far from adequate in addressing the humongous mental health burden created by the pandemic and the subsequent lockdown. As mentioned earlier, the strategy of integration of mental health into the public health system was already under struggle prior to COVID-19. Having diverted majority of the public health resources to COVID-19 response, left those in need of mental health services, in lurk.

Given the vast need for psycho-social support, many civil society organisations, groups of mental health professionals as well individuals, stepped up to the challenges and started new initiatives (Manickam 2020) . Initially, mental health services provided by civil society organisations were adversely impacted due to the lockdown and mobility restrictions. Mental health professionals, traditionally trained in face to face intervention modalities too, took some time to transition into technology assisted modalities. But soon these services were made available using technology assisted mediums; thus, bringing mental health from the shadowland into mainstream territory. These individuals, professionals and organisations dedicatedly responded to the mental health needs of the communities either by revamping or scaling up existing interventions, or by designing new ones.

And yet, the crisis created by the pandemic, made some gaps in the mental health and psychosocial responses, evident. While serving as an important resource, some of these mental health initiatives, were often informed by the logic of 'urgency'; lacking a comprehensive framing of the issue, as well as a cohesive vision. Despite some of the guiding frameworks laid down for provision of teletherapy (NIMHANS 2020), the novelty and excitement around the medium of technology, in some cases, overshadowed core issues such as conceptualisation of mental health (or the response) and training, quality, sensitivity, ethics and care and support for the volunteers and the professionals who provided these services. The work of the volunteers/professionals sometimes remained unsupervised and unsupported, leading to great amounts of burn out, as many of them struggled with similar issues as those of their clients. Newspapers carried reports of non-responsive helplines where many (especially those who belonged to the vulnerable groups such as migrant workers, students, tourists and others confined due to the lockdown) reached out for help; only to be met with by no replies (Jaiswal 2020). It was also unfortunate to see commercialisation of mental health services during the pandemic at the hands of private players.

The psycho-social nature of the impact necessitated a psycho-social and integrated response. Despite that, some of the mental health initiatives continued to view this impact and its consequent response within the confines of the traditional 'diagnostic', 'individualistic', and 'psychological' models. The distress experienced by people was reduced to a 
discrete set of symptoms when looked at through a diagnostic lens. The linkages between the individual, interpersonal and structural determinants of mental health were often missing in many of these responses.

This (as well as the concerns reported by iCALL's callers) once again, necessitates us to look at a psycho-social conceptualisation of distress, which appreciates the interconnectedness and interplay of factors situated at various levels of ecology. It is clear that the framing of the problem as well as that of the solutions, needs to be cognizant of this complexity. Discourse of mental illness is known to flatten identities by reducing it to biology or symptoms. Psycho-social discourse on the other hand brings out the textures of complex lived realities. The conceptualisation of distress and mental health concerns in psycho-social approach, is situated at various levels of ecological analysis, and so are the corresponding interventions. These interventions thus, simultaneously address the distress experienced by individuals and communities as well as the interpersonal, societal and structural determinants of this distress. The framing of distress using psycho-social lens provides directions for planning future mental health as well as development, relief and social protection measures.

\section{What is needed: directions for future}

The mass level psycho-social effects of the pandemic, while devastating and challenging, have provided us with an opportunity to bring issues of psychological distress and mental health out of the shadowland. This has also offered us an opportunity to look at the possible ways of intervening with these effects during the pandemic, and planning beyond the pandemic by building a robust psychosocial care system for future.

There is a need for building an integrated and coordinated response to deal with the psychosocial impact of the pandemic, as there are multiple sources of stressors that are simultaneously experienced by the individuals — some due to the pandemic; others due to the containment strategies and the heightening of pre-existing vulnerabilities or of socioeconomic fissures within the Indian society. Hence, it is important to follow a "whole-ofsociety" approach. This approach emphasizes the significant roles played by all sectors of society such as national government (communication and overall coordination, putting in place the necessary legislation, policies and resources, capacity development etc.), the health sector (health, mental health and psycho-social service delivery), non-health sectors (essential operations and services to mitigate health, economic and social impacts), civil society organizations (raise awareness, communicate accurate information, provide relief services, liaise with the government etc.) and families and individuals (reduce the spread of pandemic through adoption of preventive measures, and providing care and support) (WHO 2010a, b). Psychosocial components should be integrated into the National health emergency response planning, general healthcare delivery and also in other preexisting structures in the community, such as schools, community centres, youth and senior centres etc.

The following section elaborates upon a few of the specific measures that can be adopted for an effective response to the psychosocial impact of the pandemic. Needless to say, that the list is merely illustrative, and may not be completely exhaustive. Some of these interventions have been drawn from the documents designed to guide psychosocial interventions during emergencies and pandemics (e.g. IASC Guidelines on Mental Health and Psychosocial Support in Emergency Settings 2007; IASC guide on Mental Health and 
Psychosocial Support in Ebola Virus Disease Outbreaks 2015; WHO guiding document on Pandemic Influenza preparedness and Response 2010a, b).

\section{Ongoing provision of reliable information about health and preventive measures}

- Provision of reliable information on the risk, severity, and progression of a pandemic and the effectiveness of interventions used during a pandemic on an ongoing basis (WHO 2010a, b). It should also contain the exact recommended prevention methods of reducing risk, and the availability of medical evaluation and treatment, and how and where to obtain these. Given the low levels of awareness in the initial phases of pandemics, communities need reliable and simplified information. In a country like India, the information should be made available in an accessible manner (e.g. also to those who are not literate, have sensory disabilities etc.) in multiple languages.

- Ongoing communication about measures taken by the government to contain the spread of virus, and its subsequent impacts on mobility, transportation, educational institutions, workplaces, industry, etc.

- Provision of effective and ongoing teleconsultation/telephone helpline services in multiple languages for individuals to seek information and ask queries regarding health concerns as well as those related to Government guidelines, testing facilities, health services, transportation facilities and relief work including food, shelter, financial support etc. Information about these helpline or tele consultation facilities should be regularly published for people to know about these services. The services should be provided using technologies that are available and affordable to masses. Guidelines for providing technology assisted psycho-social support should be available.

- Brief, non-sensationalistic, non-blaming media messaging and IEC (information, education and communication) material for public education that emphasises positive but realistic expectations of hope, resilience and recovery.

\section{Human resource development and training}

- Developing different cadres of psychosocial care providers who offer support to individuals and communities. These include trained mental health professionals, community workers, volunteers, family members, self-help group members etc.

- Building capacities of professionals and staff working with other systems such as schools, institutions, child protection system, youth development programmes, women's organisations, relief work etc, in providing psychosocial support.

- Enhancing capacities of existing mental health care professionals in disaster and crisis counselling. Given the mobility restrictions and shift to online modes of service provision, mental health care professionals also need to receive training and support for the same. This involves building competencies related to technology, adaptation of psychosocial interventions to technological formats, knowledge about ethical norms, and handling crisis and managing safety concerns in technology assisted formats. 
- Integrating topics of disaster and crisis counselling in training curriculum for health and mental health professionals. Similarly, it is important to build components of technology-assisted counselling and interventions in mental health curricula.

- Integrating psychosocial perspective and skills into the training of counsellors working with other government programmes such as Rashtriya Kishore Swasthya Karykram (RKSK, adolescent health programme), School Health programme, One-stop crisis centres for women facing violence, health counselling in hospital setting etc.

- Providing training in psychosocial care to health care providers, frontline workers as well as those who are involved in relief work, and also providing guidelines and protocols for integrating and implementing this care.

- Supervision and support for psychosocial and mental health care providers since many of them are working in highly resource constrained environments, and are going through similar traumatic events as those of their clients.

\section{Provision of diverse psycho-social and mental health support options}

- Creation of awareness of psychosocial impact of the pandemic, and of tools for selfcare (on issues such as well-being, sleep, exercise, media consumption, managing work, handling difficult emotions, parenting, addressing relational conflicts, staying safe etc) and for supporting others (how to talk about COVID-19 to children, how to support elderly, or those who are grieving or are in distress etc.). Letting individuals know that distress is a normal reaction to an abnormal situation, and that it is okay to talk about it is important. Using effective media to communicate these messages to general public is also equally important. Additionally, it is helpful to provide possible suggestions for managing anxiety that may seem realistic during crisis or emergency situations, by adopting measures such as limiting media exposure, referring to only trusted sources of information, focusing on factors within one's control, suggesting body/breathing based methods to contain anxiety etc.

- Universal psychosocial care and support to address the needs of all potentially affected members of the community. This requires compilation of an updated list or directories of available psychosocial services.

- Psychosocial, mental health and relief related services, available to all, without any discrimination on the basis of social locations such as caste, gender, religion, sexuality, disability etc.

- Services that are not merely diagnostic and guided by large psychiatric institutions, but are community-based. These services should view distress as a normal response to an abnormal external traumatic event, and should help people enhance individuals' coping using existing strengths, skills, abilities, competencies, life philosophies and support systems. The distress also needs to be viewed in the context of structural inequalities.

- Making available an array of psychosocial support services that are accessible and culturally sensitive, ranging from counselling services, support groups, shelter homes, community-based support mechanisms, relief measures, protection services, legal aid, financial aid, livelihood options etc. and emergency mental health services for a small minority who may need it due to the serious impact of the pandemic or due to exacerbation of pre-existing mental health vulnerabilities. These services should be made available both in an online/helpline (or technologically assisted) as well as offline manner to improve accessibility. 
- Equipping individuals and communities with information and tools for addressing relational concerns and conflicts, and providing spaces to draw external support in cases where individuals are feeling lonely, isolated or distressed due to relational triggers.

- Provision of services which are integrated, and which include not only psychosocial care, but also other support services such as shelter, food, transportation, livelihood options, educational and skill building opportunities etc.

- Special consideration to psychosocial services and support structures for vulnerable and disadvantaged groups such as those who are diagnosed with COVID-19, family members who are grieving for their losses, women, LGBTQ individuals, persons with disability, individuals with prior history of mental illness etc. Service providers must take special efforts to reach out to the vulnerable groups, and also to familiarise themselves with their worlds. The context of inequalities, discrimination and injustices faced by these individuals has to be kept in mind while understanding their distress and providing services.

- Service provision for women and girl survivors of violence and abuse (helplines, safety and protection mechanisms, judicial and criminal justice system-based interventions) in the light of the growing incidence of violence and abuse against women and girls during the pandemic.

- Special considerations are also needed to address psychosocial impact of the pandemic on healthcare providers and frontline workers.

- Although not included in the group of vulnerable populations, adolescents and young individuals too need support in navigating through online education, career shifts and dwindling work opportunities due to the economic crisis created by the pandemic.

\section{Rebuilding community life, networks and society}

- Providing self-care tools for children, adolescents, youth, elderly women, etc. to manage their distress and to build resilience.

- Equipping community members with skills for identifying signs of distress and providing support.

- Creating (offline or online) spaces and opportunities for family and community members (as well as those who were diagnosed with COVID-19) to connect to each other and draw support.

- Facilitating the creation of inclusive, community-based self-help support groups for generating mutual support, fostering problem sharing, brainstorming on solutions or finding more effective ways of coping.

- Encouraging conversations on psychosocial distress within organisations and workspaces; training those in leadership positions in psychosocial care provision and building organisational measures and policies for employee well-being.

- Working towards reduction of prevalent prejudices and stigma in communities

- Finally, it is important to look at not just individual and community networks, but also at the pre-COVID political and economic contexts that have produced the suffering, especially among those who belong to the disadvantaged sections of the society. These political, social and economic contexts need to be addressed for building of new future. 


\section{Conclusion and way forward}

To begin with, there is an urgent need for a conscious framing and inclusion of psycho-social response in designing of interventions during COVID-19 pandemic across sectors (social security, housing, livelihood, education, women's empowerment etc.), and for prioritising this response. There is a need for a dialogue among health, relief, economic and mental health measures. The psycho-social response needs to simultaneously address the psychological distress experienced by individuals and communities, and also the structural triggers for the same. The latter requires immediate action in terms of provision of food, shelter, livelihood, safety etc., as well as long-term measures for addressing pre-existing health inequalities and economic and social disparities that have got further intensified during the pandemic. This necessitates being cognizant of the needs of those, who belong to the vulnerable sections of the society. It also requires considerations of social identities around caste, class, gender, sexuality etc. in influencing mental health and responses to address the same.

Mental health is a development issue. There exists a reciprocal relationship between mental health and development, with one affecting the other. Areas of development, such as education, employment, economic resources, emergency responses and human rights, impact mental health. At the same time, People with mental health concerns are often missed, or are actively excluded by development programmes. Hence it is important to improve development contexts of communities and also to address issues of individuals with mental health concerns in development interventions. Governments, civil society, bilateral development agencies, research institutions and others need to make conscious efforts to reach out to people with psycho-social disabilities (WHO 2010a, b).

The importance of technology in creating mental health awareness, in building capacities of mental health personnel and in provision of services, has stood out as a significant outcome of the pandemic. On one hand, it is important to explore the multiple possibilities of reach and creativity that technology holds for the future; but on the other, it is also necessary to be not lured by the projections of technology as a panacea for all the mental health problems. We must remember that access to technology is often mediated by power hierarchies within the Indian households. Besides, the Indian society largely interacts through face-to-face encounters. Hence a combination of 'online' and 'offline' solutions is necessary for mental health services to be viable, acceptable and sustainable in India. We also need to invest in and nurture the human resources that make this technology alive.

There is a need for creating use-friendly resources that de-mystify mental health and mitigate the stigma around it. It is important to include community in voicing their needs and designing and implementing interventions. There are numerous instances during the pandemic, where it was the support of the community and neighbourhoods that helped people navigate through the challenges faced. Involvement of the community would ensure entire society's recovery. Finally, there is a need for investing in building an effective psycho-social and mental health infrastructure (United Nations 2020). It is also time to invest in creation of human resources and enhancing capacities of the existing ones. These resources and services, if consciously created, can survive beyond the pandemic, and continue to serve as a vital resource for communities. COVID-19 pandemic, while devastating in its impact, has provided opportunities for creating awareness and resources for psychosocial and mental health care in India. 


\section{References}

Alegría M, NeMoyer A, Falgàs Bagué I, Wang Y, Alvarez K (2018) Social determinants of mental health: where we are and where we need to go. Curr Psychiatry Rep 20(11):95. https://doi. org/10.1007/s11920-018-0969-9

Bannerjee D (2020) Letter to the editor: the impact of Covid-19 pandemic on elderly mental health. Int J Geriatr Psychiatry 2020:1-2

Bowen M (1978) Family therapy in clinical practice. Jason Aronson Inc, Northvale

Braun V, Clarke V (2006) Using thematic analysis in psychology. Qual Res Psychol 3(2):77-101

Bronfenbrenner U (1986) Ecology of the family as a context for human development: research perspectives. Dev Psychol 22(6):723-742

Burgess R (2020) COVID-19 mental-health responses neglect social realities. Nature. https://doi. org/10.1038/d41586-020-01313-9

Burns J (2016) Psychosocial determinants of mental disorders. In: Textbook of psychiatry for southern Africa, 2nd edn. Oxford University Press, Cape Town

Chatterjee K, Chauhan VS (2020) Epidemics, quarantine and mental health. Med J 76(2):125-127. https ://doi.org/10.1016/j.mjafi.2020.03.017

Chatterjee P (2020) Gaps in India's preparedness for COVID-19 control. Lancet Infect Dis 20(5):544

Chatterjee S, Bhattacharyya R, Bhattacharyya S, Gupta S, Das S, Banerjee B (2020) Attitude, practice, behavior, and mental health impact of COVID-19 on doctors. Indian J Psychiatry 62(3):257-265

Choudhari R (2020) COVID 19 pandemic: mental health challenges of internal migrant workers of India. Asian J Psychiatry 54:102254. https://doi.org/10.1016/j.ajp.2020.102254

Council of Europe (2020) Statement by human rights experts on the International Day against Homophobia, Transphobia and Biphobia. COVID-19: The suffering and resilience of LGBT persons must be visible and inform the actions of States. https://www.coe.int/en/web/commissioner/-/covid -19-the-suffering-and-resilience-of-lgbt-persons-must-be-visible-and-inform-the-actions-of-states

Engel G (1977) The need for a new medical model: a challenge for biomedicine. Science 196(4286):129-136

Grace SL, Hershenfield K, Robertson E, Stewart DE (2005) The occupational and psychosocial impact of SARS on academic physicians in three affected hospitals. Psychosomatics 46(5):385-391. https://doi. org/10.1176/appi.psy.46.5.385

Gupta S, Sagar R (2018) National mental health programme-optimism and caution: a narrative review. Indian J Psychol Med 40(6):509-516. https://doi.org/10.4103/IJPSYM.IJPSYM_191_18

Hatzenbuehler M, Phelan J, Link B (2013) Stigma as a fundamental cause of population health inequalities. Am J Public Health 103:813-821

Huang Y, Zhao N (2020) Mental health burden for the public affected by the COVID-19 outbreak in China: who will be the high-risk group? Psychol Health Med. https://doi.org/10.1080/13548506.2020.17544 38

Inter-Agency Standing Committee Reference Group on Mental Health and Psychosocial Support in Emergency Settings (2015) Mental Health and Psychosocial Support in Ebola Virus Disease Outbreaks: A Guide for Public Health Programme Planners. Geneva

Inter-Agency Standing Committee, I.-A. S. (2007) IASC guidelines on mental health and psychosocial support in emergency settings. Author, Geneva

IOM (2020) Mental health and psychosocial support (MHPSS) in the COVID-19 response: guidance and toolkit for the use of IOM MHPSS teams: version III-final. IOM, Geneva

Jacob K (2006) Social context and mental health, distress and illness: critical yet disregarded by psychiatric diagnosis and classification. Indian J Soc Psychiatry 32(3):243-248

Jain S, Jadhav S (2009) Pills that swallow policy: clinical ethnography of a community mental health program in northern India. Transcult Psychiatry 46(1):60-85

Jaiswal B (2020) Unhelpful helplines in times of COVID-19. Downloaded from https://www.theweek.in/ news/india/2020/04/30/unhelpful-helplines-in-times-of-covid-19.html

Johal S, Macdonald C, Mounsey Z (2016) Framework for psychosocial support in emergencies. Ministry of Health, New Zealand

John A (2020) Supporting students and the school community during a pandemic. In: Manickam LSS (ed) COVID-19 pandemic: challenges and responses of psychologists from India. The Editor, Thiruvananthapuram, pp 56-62

John C, Gunasekaran I, Maria R (2020) COVID-19: taking heed from Kerala's mental health interventions. Sci Wire https://science.thewire.in/health/covid-19-kerala-mental-health/

Kasthuri A (2018) Challenges to healthcare in India-the five A's. Indian J Community Med 43(3):141143. https://doi.org/10.4103/ijcm.IJCM_194_18 
Ko C-H, Yen Ju-Yu, Yang M-J (2006) Psychosocial impact among the public of the severe acute respiratory syndrome epidemic in Taiwan. Psychiatry Clin Neurosci 60:397-403. https://doi.org/10.111 1/j.1440-1819.2006.01522.x

Kumar S, Jayraman S, Rangaswamy K (2020) Tamil Nadu Association of clinical psychologists (TNACP) responding to COVID-19 pandemic. In: Manickam LSS (ed) COVID-19 pandemic: challenges and responses of psychologists from India. The Editor, Thiruvananthapuram, pp 204-208

Macneil C, Hasty M, Conus P, Berk M (2012) Is diagnosis enough to guide interventions in mental health? Using case formulation in clinical practice. BMC Med 10:111. https://doi. org/10.1186/1741-7015-10-111

Malathesh BC (2020) Response to: rethinking online mental health services in China during the COVID19 epidemic. Asian J. Psychiatr. https://doi.org/10.1016/j.ajp.2020.102105

Manickam SL (2020) COVID- 19 pandemic: challenges and responses of psychologists from India. Centre for Applied Psychological Studies, Thiruvananthapuram

Mathews R (2020) Covid-19: why are women more vulnerable to mental health issues? https://femin isminindia.com/2020/05/05/covid-19-women-vulnerable-mental-health-issues/

Ministry of Health and Family Welfare (2020) Behavioural health: psycho-social toll-free helpline. https ://www.mohfw.gov.in/

Moses N (2020) Covid-19: India is staring at a mental health crisis. Hindustan Times, https://www. hindustantimes.com/analysis/covid-19-india-is-staring-at-a-mental-health-crisis/story-hmBOz UYsbo3SmtlWilmBzL.html

Nair L (2020) Battling mental health impact of COVID-19. Downloaded from https://www.expresshea lthcare.in/covid19-updates/battling-mental-health-impact-of-covid-19/424295/

National Institute of Mental Health and Neuro Sciences (2020) Guidelines for tele-psychotherapy services

National Institute of Mental Health and Neuro Sciences (2016) National Mental Health Survey of India, 2015-16: Summary. NIMHANS Publication No, Bengaluru, p 128

PAHO/WHO (2016). Mental health protection and psychosocial support in pandemic settings. Downloaded from https://www.paho.org/disasters/index.php?option=com_docman\&view=downl oad\&alias =2542-mental-health-protection-psychosocial-support-epidemic-settings-2016$542 \&$ category_slug $=$ mental-health \&Itemid=1179\&lang $=$ en

Pandey R, Kukreja S, Kumar R, Priya KR (2020) Covid-19: mental healthcare without social justice? Econ Polit Wkly 55:31

Kinderman P (2014) A prescription for psychiatry: why we need a whole new approach to mental health and wellbeing, 1st edn. Palgrave Macmillan, London

Priya KR (2018) Well-being and mental health in the aftermath of disasters: a social constructionist approach. https://doi.org/10.1007/978-81-322-3782-2_23

Raghavan R, Cooper J (2018) Mental health of internal migrants in India: an examination of current strengths and gaps in current data. Eur J Public Health 28(Suppl_1), cky048.214. https://doi. org/10.1093/eurpub/cky048.214

Rajkumar R (2020) COVID-19 and mental health: a review of the existing literature. Asian J Psychiatr 2020(52):102066. https://doi.org/10.1016/j.ajp.2020.102066

Rana U (2020) Elderly suicides in India: an emerging concern during COVID-19 pandemic. Int Psychogeriatrics. https://doi.org/10.1017/S1041610220001052

Roesch E, Amin A, Gupta J, García-Moreno C (2020) Violence against women during COVID-19 pandemic restrictions. BMJ 369:m1712. https://doi.org/10.1136/bmj.m1712

Rosen CS, Glassman LH, Morland LA (2020) Telepsychotherapy during a pandemic: a traumatic stress perspective. J Psychother Integr 30(2):174-187. https://doi.org/10.1037/int0000221

Roy E (20203). Domestic violence, abuse complaints rise in coronavirus lockdown: NCW. The Indian Express. Retrieved from https://indianexpress.com/article/india/ domestic-violence-abuse-complaints-rise-in-coronavirus-lockdown-ncw-6344641/

Sahoo H, Biswal R (2020). Impact of Covid 19 on mental health: whether India is prepared to handle the crisis? Online at https://mpra.ub.uni-muenchen.de/100765/. MPRA Paper No. 100765, posted 05 Jun 2020 17:23 UTC

Singh R, Subedi M (2020) COVID-19 and stigma: social discrimination towards frontline healthcare providers and COVID-19 recovered patients in Nepal. Asian J Psychiatry 53:102222. https://doi. org/10.1016/j.ajp.2020.102222

Spoorthy MS, Pratapa SK, Mahant S (2020) Mental health problems faced by healthcare workers due to the COVID-19 pandemic_a review. Asian J Psychiatry 51:102119. https://doi.org/10.1016/j. ajp.2020.102119 
Sudan P, Narayan K (2020) In India, the deepening of the mental health crisis IOpinion. Hindustan Times. https://www.hindustantimes.com/analysis/in-india-the-deepening-of-the-mental-health-crisis/story -lsi7m4qbPlQOFbv9qRhkvL.html

Sytema S (2006) Mental health at the crossroads: the promise of the psychosocial approach. Int J Integr Care. http://doi.org/10.5334/ijic. 149

United Nations (2020) Policy brief: Covid-19 and the need for action on mental health. https://unsdg.un.org/ sites/default/files/2020-05/UN-Policy-Brief-COVID-19-and-mental-health.pdf

Van Bortel T, Basnayake A, Wurie F, Jambai M, Koroma A, Muana A, Hann K, Eaton J, Martin S, Nellums L (2016). Psychosocial effects of an Ebola outbreak at individual, community and international levels. Bull World Health Organ. https://doi.org/10.2471/blt.15.158543

Varshney M, Thomas PJ, Raizada N, Sarin S (2020) Initial psychological impact of COVID-19 and its correlates in Indian Community: an online (FEEL-COVID) survey. PLoS ONE. https://doi.org/10.1371/ journal.pone. 0233874

Veliyannoor PV (2020) The return of the repressed. In: COVID-19: the need for intervention at socio-cultural inscape. In: Manickam LSS (ed) COVID-19 pandemic: challenges and responses of psychologists from India. The Editor, Thiruvananthapuram, pp 108-129

Ventevogel P (2018) Interventions for mental health and psychosocial support in complex humanitarian emergencies: moving towards consensus in policy and action? Theory, research and clinical practice. https://doi.org/10.1007/978-3-319-97046-2_8

Weir K (2020) Seven crucial research findings that can help people deal with COVID-19. http://apa.org/ news/apa/2020/03/covid-19-research-findings

Weiss MG, Saraceno B, Saxena S, van Ommeren M (2003) Mental health in the aftermath of disasters: consensus and controversy. J Nerv Ment Dis 191(9):611-615. https://doi.org/10.1097/01.nmd.00000 87188.96516.a3

World Health Organisation (2009) Pandemic influenza: preparedness and Response: a WHO guidance document

World Health Organisation (2010) Mental health and development: targeting people with mental health conditions as a vulnerable group

World Health Organization (2014) Social determinants of mental health. World Health Organization. https:// apps.who.int/iris/handle/10665/112828

World Health Organization (2001) Mental health problems: the undefined and hidden burden. https://www. who.int/mediacentre/

Yeung J, Gupta S (2020) Doctors evicted from their homes in India as fear spreads amid coronavirus lockdown, CNN. https://edition.cnn.com/2020/03/25/asia/india-coronavirus-doctors-discrimination-intlhnk/index.html

Zacharias L, Harikrishnan U, Jayakumar C, Sekar K (2019) Psychosocial perspective of Nipah virus outbreak in Kerala, India. https://doi.org/10.17354/ijss/2018/30

Zhou J, Liu L, Xue P, Yang X, Tang X (2020) Mental health response to the COVID-19 outbreak in China. Am J Psychiatry 177(7):574-575. https://doi.org/10.1176/appi.ajp.2020.20030304

Publisher's Note Springer Nature remains neutral with regard to jurisdictional claims in published maps and institutional affiliations. 